\title{
A Method for the Computation of the Fresnel Integrals and Related Functions
}

\author{
By Oscar L. Fleckner*
}

Abstract. A new method for computing the values of the Fresnel integrals and related functions is developed. Error estimates for the resulting power series are derived, and an application of the technique is discussed.

1. Introduction. The objective of this paper is to develop a method for computer generation of the functions $C(x, \alpha)$ and $S(x, \alpha)$ where

$$
\begin{array}{ll}
C(x, \alpha)=\int_{0}^{x} \cos t / t^{\alpha} d t & (0 \leqq \alpha<1), \\
S(x, \alpha)=\int_{0}^{x} \sin t / t^{\alpha} d t & (0 \leqq \alpha \leqq 1)
\end{array}
$$

for $x \geqq 0$.

Note that $S(x, 1)$ is the familiar sine integral $S i(x)$. Also for $\alpha=1 / 2$ we have

$$
(2 \pi)^{-1 / 2} C(x, 1 / 2)=C(x) \text { and }(2 \pi)^{-1 / 2} S(x, 1 / 2)=S(x),
$$

where $C(x)$ and $S(x)$ are the well-known Fresnel integrals (see [5]). Often in the literature $C\left(\pi x^{2} / 2\right)$ and $S\left(\pi x^{2} / 2\right)$ are called Fresnel integrals and are denoted by $C(x)$ and $S(x)$, respectively, (see [1]).

2. Analysis. The Maclaurin expansions for $C(x, \alpha)$ and $S(x, \alpha)$ are given by

$$
\begin{aligned}
& C(x, \alpha)=x^{(1-\alpha)} \sum_{\nu=0}^{\infty} \frac{(-1)^{\nu} x^{2 \nu}}{(2 \nu+1-\alpha)(2 \nu) !}, \\
& S(x, \alpha)=x^{(2-\alpha)} \sum_{\nu=0}^{\infty} \frac{(-1)^{\nu} x^{2 \nu}}{(2 \nu+2-\alpha)(2 \nu+1) !} .
\end{aligned}
$$

By the ratio test one can show that these series converge for all real values of $x$, although it is clear that for large $x$ many terms are required before succeeding terms become negligible.

For relatively small values of $x$ a digital computer program that evaluates the truncated power series for $C(x, \alpha)$ and $S(x, \alpha)$ directly will produce excellent results both in accuracy and speed. It is also evident that for large values of $x$, use of the power series technique will lead to computational difficulties such as "roundoff" error and loss of significance due to the large number of terms needed to insure a specified accuracy. Hence, an alternative computational technique is required for large values of $x$.

For $x \leqq 5 \pi / 2, C(x, \alpha)$ can be evaluated by direct substitution in its truncated

Received November 13, 1967.

* The author is currently a graduate student in the Department of Mathematics at The Ohio State University and is employed at The Ohio State University Computer Center. 
power series. Hence, let an arbitrary $x_{0}>5 \pi / 2$ be given. Let $k$ be a nonnegative integer such that $(2 k+1) \pi / 2<x_{0} \leqq(2 k+3) \pi / 2$ and put $\theta_{k}=(2 k+1) \pi / 2$. Then it readily follows that

$$
C\left(x_{0}, \alpha\right)=C\left(\theta_{k}, \alpha\right)+(-1)^{k+1} \theta_{k}{ }^{-\alpha} \int_{0}^{x_{0}-\theta_{k}}\left(1+y / \theta_{k}\right)^{-\alpha} \sin y d y .
$$

In a similar fashion, for $x_{1}>2 \pi, m$ the greatest nonnegative integer such that $m \pi<x_{1}$, and $\phi_{m}=m \pi$, we get

$$
S\left(x_{1}, \alpha\right)=S\left(\phi_{m}, \alpha\right)+(-1)^{m} \phi_{m}^{-\alpha} \int_{0}^{x_{1}-\phi_{m}}\left(1+y / \phi_{m}\right)^{-\alpha} \sin y d y .
$$

For notational simplicity, let

$$
H(\rho, \gamma, \alpha)=\int_{0}^{\rho}(1+y / \gamma)^{-\alpha} \sin y d y .
$$

Then (2.2) and (2.3) become

$$
\begin{aligned}
& C\left(x_{0}, \alpha\right)=C\left(\theta_{k}, \alpha\right)+(-1)^{k+1} \theta_{k}^{-\alpha} H\left(x_{0}-\theta_{k}, \theta_{k}, \alpha\right), \\
& S\left(x_{1}, \alpha\right)=S\left(\phi_{m}, \alpha\right)+(-1)^{m} \phi_{m}{ }^{-\alpha} H\left(x_{1}-\phi_{m}, \phi_{m}, \alpha\right) .
\end{aligned}
$$

Assuming that the values of $C\left(\theta_{k}, \alpha\right)$ and $S\left(\phi_{m}, \alpha\right)$ are known, the problem of evaluating the functions $C(x, \alpha)$ and $S(x, \alpha)$, as equations (2.5) show, has been reduced to the problem of evaluating the integral $H$. We prove the following theorem.

Theorem 1. If $0<\rho<\gamma$ and $0 \leqq \alpha \leqq 1$, and $H(\rho, \gamma, \alpha)$ is as in (2.4), then

$$
H(\rho, \gamma, \alpha)=\sum_{\nu=0}^{\infty} a_{\nu} K_{\nu}(\rho, \gamma)
$$

where

$$
\begin{array}{r}
a_{\nu}=\frac{(-1)^{\nu}}{\nu !}(\alpha)_{\nu}=\frac{(-1)^{\nu}}{\nu !} \Gamma(\alpha+\nu) / \Gamma(\alpha), \\
K_{\nu}(\rho, \gamma)=\int_{0}^{\rho}(y / \gamma)^{\nu} \sin y d y \quad(\nu=0,1, \cdots) .
\end{array}
$$
Thus,

Proof. Since $y / \gamma<1$ and $0 \leqq \alpha \leqq 1$, the expansion of $(1+y / \gamma)^{-\alpha}$ is valid.

$$
\begin{aligned}
& \int_{0}^{\rho}(1+y / \gamma)^{-\alpha} \sin y d y \\
& \quad=\int_{0}^{\rho}\left[1-\frac{(\alpha)_{1}}{1 !}(y / \gamma)+\frac{(\alpha)_{2}}{2 !}(y / \gamma)^{2}-\frac{(\alpha)_{3}}{3 !}(y / \gamma)^{3}+\cdots\right] \sin y d y .
\end{aligned}
$$

Termwise integration yields

$$
H(\rho, \gamma, \alpha)=\sum_{\nu=0}^{\infty} \frac{(-1)^{\nu}}{\nu !}(\alpha)_{\nu} K_{\nu}(\rho, \gamma),
$$

and the result is proved.

Applying Theorem 1 to the expressions for $C\left(x_{0}, \alpha\right)$ and $S\left(x_{1}, \alpha\right)$ given by equations (2.5), we obtain 


$$
\begin{aligned}
& C\left(x_{0}, \alpha\right)=C\left(\theta_{k}, \alpha\right)+(-1)^{k+1} \theta_{k}^{-\alpha} \sum_{\nu=0}^{\infty} a_{\nu} K_{\nu}\left(x_{0}-\theta_{k}, \theta_{k}\right), \\
& S\left(x_{1}, \alpha\right)=S\left(\phi_{m}, \alpha\right)+(-1)^{m} \phi_{m}^{-\alpha} \sum_{\nu=0}^{\infty} a_{\nu} K_{\nu}\left(x_{1}-\phi_{m}, \phi_{m}\right) .
\end{aligned}
$$

We have now reduced our computational problem to evaluating a sum of the form

$$
\sum_{\nu=0}^{\infty} a_{\nu} K_{\nu}(\rho, \gamma)
$$

where the changes of variable that we have performed have restricted $\rho$ such that $0 \leqq \rho \leqq \pi<\gamma$. The following theorem gives a recursion scheme for computing the values of $K_{\nu}(\rho, \gamma), \nu=0,1,2, \cdots$.

TheOREM 2. The auxiliary functions $K_{\nu}(\rho, \gamma)$ satisfy the following recurrence relation:

$$
\begin{aligned}
& K_{0}(\rho, \gamma)=1-\cos \rho \\
& K_{1}(\rho, \gamma)=(1 / \gamma) \sin \rho-(\rho / \gamma) \cos \rho \\
& K_{n}(\rho, \gamma)=c_{n} K_{n-2}(\rho, \gamma)+d_{n}, \quad n=2,3, \cdots
\end{aligned}
$$

where

$$
\begin{aligned}
& c_{n}=-n(n-1) / \gamma^{2}, \\
& d_{n}=-(\rho / \gamma)^{n} \cos \rho+(n / \gamma)(\rho / \gamma)^{n-1} \sin \rho .
\end{aligned}
$$

Proof. The expressions for $K_{0}(\rho, \gamma)$ and $K_{1}(\rho, \gamma)$ follow immediately by direct integration of (2.7). Two applications of integration by parts to (2.7) yield the desired result.

Equations (2.8) together with Theorems 1 and 2 solve the problem of the evaluation of the functions $C(x, \alpha)$ and $S(x, \alpha)$. Let us now try to estimate the rate of convergence of $(2.9)$.

THEOREM 3. If $0 \leqq \rho \leqq \pi<\gamma$, then for any positive integer $N$,

$$
\left|\sum_{n=0}^{\infty} a_{n} K_{n}(\rho, \gamma)-\sum_{n=0}^{N-1} a_{n} K_{n}(\rho, \gamma)\right| \leqq\left|a_{N} K_{N}(\rho, \gamma)\right| \leqq\left(\frac{\pi}{N+1}\right)\left(\frac{\pi}{\gamma}\right)^{N} .
$$

Proof. Since $0 \leqq \rho \leqq \pi<\gamma$, we have

$$
(y / \gamma)^{n} \sin y \geqq 0 \quad \text { for } \quad 0 \leqq y \leqq \rho .
$$

Thus, $K_{n}(\rho, \gamma) \geqq 0$ for all $n$, and

$$
K_{n}(\rho, \gamma) \leqq(1 / \gamma)^{n} \int_{0}^{\rho} y^{n} d y=\frac{\rho^{n+1}}{(n+1) \gamma^{n}} .
$$

Hence,

$$
K_{n}(\rho, \gamma) \leqq\left(\frac{\pi}{n+1}\right)\left(\frac{\pi}{\gamma}\right)^{n}
$$

and since $\left|a_{n}\right| \leqq 1$ for all $n$, we have

$$
\left|a_{n} K_{n}(\rho, \gamma)\right|=\left|a_{n}\right| K_{n}(\rho, \gamma) \leqq\left(\frac{\pi}{n+1}\right)\left(\frac{\pi}{\gamma}\right)^{n}
$$

for all $n$. Therefore, 


$$
\sum_{n=0}^{\infty}\left|a_{n} K_{n}(\rho, \gamma)\right| \leqq \pi \sum_{n=0}^{\infty}\left(\frac{1}{n+1}\right)\left(\frac{\pi}{\gamma}\right)^{n} .
$$

The latter sum of (2.14) converges by the ratio test. Therefore (2.9) converges by comparison. Applying the property of an alternating convergent series in which the terms are monotonically decreasing in magnitude yields the first inequality in (2.11). The result now follows from (2.13).

Applying Theorem 3 to the summations appearing in equations (2.8), we obtain

$$
\begin{aligned}
\left|\sum_{\nu=0}^{\infty} a_{\nu} K_{\nu}\left(x_{0}-\theta_{k}, \theta_{k}\right)-\sum_{\nu=0}^{N-1} a_{\nu} K_{\nu}\left(x_{0}-\theta_{k}, \theta_{k}\right)\right| & \leqq\left(\frac{\pi}{N+1}\right)\left(\frac{\pi}{\theta_{k}}\right)^{N} \\
& =\frac{\pi}{(N+1)(k+1 / 2)^{N}}
\end{aligned}
$$

and

$$
\begin{aligned}
\left|\sum_{\nu=0}^{\infty} a_{\nu} K_{\nu}\left(x_{1}-\phi_{m}, \phi_{m}\right)-\sum_{\nu=0}^{N-1} a_{\nu} K_{\nu}\left(x_{1}-\phi_{m}, \phi_{m}\right)\right| & \leqq\left(\frac{\pi}{N+1}\right)\left(\frac{\pi}{\phi_{m}}\right)^{N} \\
& =\frac{\pi}{(N+1) m^{N}} .
\end{aligned}
$$

3. An Application. The method derived in Section 2 of this paper for the computation of the functions $C(x, \alpha)$ and $S(x, \alpha)$ was carried out for the case of the Fresnel integrals $C(x)$ and $S(x)$ of (1.2).

In this case equations (2.8) become

$$
\begin{aligned}
& C\left(x_{0}\right)=C\left(\theta_{k}\right)+(-1)^{k+1}\left(2 \pi \theta_{k}\right)^{-1 / 2} \sum_{\nu=0}^{\infty} a_{\nu} K_{\nu}\left(x_{0}-\theta_{k}, \theta_{k}\right), \\
& S\left(x_{1}\right)=S\left(\phi_{m}\right)+(-1)^{m}\left(2 \pi \phi_{m}\right)^{-1 / 2} \sum_{\nu=0}^{\infty} a_{\nu} K_{\nu}\left(x_{1}-\phi_{m}, \phi_{m}\right) .
\end{aligned}
$$

The method itself was implemented as follows. A maximum value of the argument, $x_{\max }$, was chosen. In the case of $C(x)$, the greatest nonnegative integer $K$ was determined such that $(2 K+1) \pi / 2<x_{\max }$. Then the integrals $C\left(\theta_{k}\right)$ were computed for $k=2,3, \cdots, K$, where $C\left(\theta_{2}\right)$ was evaluated by direct substitution in the truncated power series expansion for $C(x) . C\left(\theta_{3}\right), C\left(\theta_{4}\right), \cdots, C\left(\theta_{K}\right)$ were evaluated using equation (3.1). These values were then stored in a linear array, ordered by their subscripts. In the case of $S(x)$, the greatest nonnegative integer $M$ was determined such that $M \pi<x_{\max }$. The integrals $S\left(\phi_{m}\right)$ were computed for $m=2,3, \cdots, 1 I$, where $S\left(\phi_{2}\right)$ was computed from the truncated Maclaurin expansion. $S\left(\phi_{3}\right), S\left(\phi_{4}\right), \cdots, S\left(\phi_{M}\right)$ were evaluated using (3.1). These values were also stored in an array according to their subscripts. These two tables, set up initially, were used throughout in the evaluation of $C(x)$ and $S(x)$ for various values of $x$.

Now, for $x^{\prime}<x_{\max }, C\left(x^{\prime}\right)$ and $S\left(x^{\prime}\right)$ were computed as follows. In the case of $C\left(x^{\prime}\right)$, if $0 \leqq x^{\prime} \leqq 5 \pi / 2, C\left(x^{\prime}\right)$ was evaluated by the direct substitution of $x^{\prime}$ in the truncated power series expansion for $C(x)$. If $x^{\prime}>5 \pi / 2$, the integer $k$ was determined such that $(2 k+1) \pi / 2 \leqq x^{\prime}<(2 k+3) \pi / 2$. Then from the previously computed value of $C\left(\theta_{k}\right), C\left(x^{\prime}\right)$ was determined from (3.1). For $S\left(x^{\prime}\right)$, if $0 \leqq x^{\prime} \leqq 2 \pi$, the power series technique was employed. If $x^{\prime}>2 \pi$, the integer $m$ was found 
such that $m \pi \leqq x^{\prime}<(m+1) \pi$ and since $S\left(\phi_{m}\right)$ is known, $S\left(x^{\prime}\right)$ can also be evaluated using (3.1).

4. Results. The values of $C(x)$ and $S(x)$ were computed for $x=0(.2) 60$ for purposes of checking the method and program. $x_{\max }$ was chosen to be 100 , and the program tolerance for the computation of $C\left(\theta_{k}\right)$ and $S\left(\phi_{k}\right), \quad k=2,3, \cdots, 32$ was $\pm 10^{-8}$. In the computation of $C(x)$ and $S(x)$ for $x=0(.2) 60$ a tolerance of $\pm 10^{-6}$ was chosen. The results of the computation are on deposit in the Unpublished Mathematical Tables file.

The computed values of $C(x)$ and $S(x)$ were compared with those compiled by T. Pearcey [6]. In all cases, a deviation of not more than two units in the sixth decimal place was observable.

The program, written in OSU SCATRAN (a compiler language similar to $M A D$ and $\mathrm{PL} / \mathrm{I}$ ), is available on request from the author. The computations were done on an IBM 7094 computer in single precision (i.e. 8 or 9 significant digits).

Table 1 shows the number of terms, $N$, that had to be included in order to achieve the desired accuracy in the computation of $C\left(\theta_{k}\right)$ and $S\left(\phi_{k}\right)$ for some representative values of $k$.

\begin{tabular}{|c|c|c|}
\hline$k$ & $\begin{array}{l}\text { A ABLE } \\
N(C)\end{array}$ & $N(S)$ \\
\hline$\overline{3}$ & 15 & 18 \\
\hline 6 & 9 & 9 \\
\hline 9 & 7 & 8 \\
\hline 12 & 7 & 7 \\
\hline 15 & 6 & 6 \\
\hline 25 & 5 & 5 \\
\hline 30 & 5 & 5 \\
\hline
\end{tabular}

5. Discussion. In connection with Theorem 2, it should be noted that the computational results might be enhanced by using the recurrence relation in the backward direction (see [3]).* Let $K_{n}(\rho, \gamma)$ be computed for $n=0,1, \cdots, N$ by forward recurrence (2.10), yielding $\left\{y_{n}\right\}$. Suppose that initial errors $\epsilon_{0}$ and $\epsilon_{1}$ are introduced (due to rounding, for example) and that the succeeding calculations are performed with infinite precision, i.e. we have

$$
y_{0}=K_{0}+\epsilon_{0}, \quad y_{1}=K_{1}+\epsilon_{1} .
$$

Then, for $n$ even, the relative error of $y_{n}$ is

$$
\left|\left(y_{n}-K_{n}\right) / K_{n}\right|=\left[n ! / \gamma^{n} K_{n}\right]\left|\epsilon_{0}\right| .
$$

Similarly, in case $n$ is odd,

$$
\left|\left(y_{n}-K_{n}\right) / K_{n}\right|=\left[n ! / \gamma^{n-1} K_{n}\right]\left|\epsilon_{1}\right| .
$$

Thus, from (2.12) and the fact that $\gamma>\pi$, we get

$$
\left|\left(y_{N}-K_{N}\right) / K_{N}\right| \geqq\left[(N+1) ! / \pi^{N+1}\right]|\epsilon|,
$$

where $|\epsilon|=\min \left(\left|\epsilon_{0}\right|,\left|\epsilon_{1}\right|\right)$.

* The author is indebted to the referee for pointing out this possibility. 
(5.1) shows that the straightforward application of the recurrence relation (2.10) in the forward direction may lead to serious problems depending on how many terms of (2.9) are needed to insure the specified accuracy. This suggests that in some circumstances it would be best to use (2.10) in the backward direction.

Acknowledgements. The author wishes to express his gratitude to Drs. R. Bojanic, T. W. Hildebrandt, and J. Brann for their many helpful suggestions. He is also indebted to the referee for suggesting many improvements, both in form and substance. Also, the author wishes to express his appreciation to Dr. Roy F. Reeves, Director of The Ohio State University Computer Center, for the use of the facilities during the preparation of this paper.

The Ohio State University

Columbus, Ohio

1. M. Abramowitz \& I. A. Stegun, (Editors), Handbook of Mathematical Functions with Formulas, Graphs, and Mathematical Tables, Department of Commerce, Nat. Bur. Standards Appl. Math. Series, 55, U. S. Government Printing Office, Washington, D. C., 1964; 3rd printing, with corrections, 1965. MR $29 \# 4914$; MR $31 \# 1400$.

2. R. Courant, Differential and Integral Calculus, Vols. I, II, Interscience, New York, 1960.

3. W. GAUTSCHI, "Computational aspects of three-term recurrence relations," Siam Rev., v. 9, 1967, pp. 24-82.

4. C. D. Hodgman, C. R. C. Standard Mathematical Tables, Chemical Rubber Publishing Co., Cleveland, Ohio, 1959.

5. E. JahnKe, F. Emde \& F. Lösch, Table of Higher Functions, 6th ed., McGraw-Hill, New York; Teubner, Stuttgart, 1960. MR 22 \#5140.

6. T. Pearcey, Table of the Fresnel Integrals, Commonwealth Scientific and Industrial Research Organization, Melbourne, Australia, 1956.

7. SCATRAN Reference Manual, Numerical Computation Laboratory, The Ohio State University, 1964. 